nanobody) and $\beta$ arrs, a regulator of GPCR signaling, to selected class A GPCRs has been documented recently ${ }^{12,13}$. Such complexes are referred to as super complexes, and they are proposed to be responsible for sustained cAMP generation from endosomal compartments. In such super complexes, the $G \beta \gamma$ subunits remain bound to the receptor- $\beta$ arr complex even after dissociation of the Gos subunit ${ }^{12}$. It would be interesting to explore whether class B GPCRs can also form such super complexes, and if they do, whether this interaction of helix8-G $\beta 1$ might help tether the complex. Interestingly, the parathyroid hormone receptor, another class B GPCR, not only exhibits sustained cAMP response, even after internalization, but also forms a receptorG $\beta \gamma$ - $\beta$ arr complex ${ }^{14}$.

Since the first crystal structure of the $\beta_{2} \mathrm{AR}$ was determined in 2007, close to 100 crystal structures of nearly 30 different GPCRs have been solved. Consistent with the conserved architecture, signaling and regulatory mechanisms of GPCRs, many of the methodological breakthroughs pioneered with $\beta_{2} \mathrm{AR}$ (such as fusion-protein and nanobody approaches) have been by and large directly transferrable to other GPCRs ${ }^{2,15}$. Although stable assembly and structural characterization of GPCR signaling complexes present very different challenges compared to the characterization of isolated receptors, the current cryo-EM breakthrough with the CTR-G-protein complex promises exciting prospects over the next few years. A major challenge that still remains is to capture distinct conformations of receptor-G-protein complexes during various steps of nucleotide exchange. It is also interesting that some GPCRs have strict preferences for specific $G \alpha$ subtypes, whereas others can couple to two or even more subtypes of $\mathrm{G} \alpha$. Investigating the structural basis for such preference and promiscuity of G-protein coupling depending on the context (for example, cell or ligand dependent) is likely to be a challenging but rewarding avenue to explore in order to better understand the activation and signaling of GPCRs.

What are the next structural revelations in the field of GPCRs? The structure of a GPCR-GRK (GPCR kinase) complex? The structure of a receptor-transducer-effector complex? Or the structure of a dimeric class C GPCR? Whichever of these it may be, it is likely to take us a step closer to a better understanding of GPCR signaling and regulatory paradigms with direct implications for novel drug discovery. So, stay tuned!
ACKNOWLEDGMENTS

The research program in A.K.S.'s laboratory is supported by the Wellcome Trust DBT India Alliance (IA/I/14/1/501285), the Department of Biotechnology (DBT), the Department of Science and Technology (DST), the Council for Scientific and Industrial Research (CSIR) and the Institute of Technology, Kanpur.

\section{COMPETING FINANCIAL INTERESTS}

The authors declare no competing financial interests.

1. Bockaert, J \& Pin, J.P EMBO J. 18, 1723-1729 (1999).

2. Ghosh, E., Kumari, P., Jaiman, D. \& Shukla, A.K. Nat Rev. Mol. Cell Biol. 16, 69-81 (2015).

3. Ghosh, E., Nidhi, K. \& Shukla, A.K. Cell 159 1712-1712. e1 (2014).

4. Rasmussen, S.G. et al. Nature 477, 549-555 (2011).

5. Kang, Y. et al. Nature 523, 561-567 (2015).

6. Westfield, G.H. et al. Proc. Natl. Acad. Sci. USA 108 , 16086-16091 (2011).

7. Shukla, A.K. et al. Nature 512, 218-222 (2014)

8. Liang, Y.L. et al. Nature http://dx.doi.org/10.1038/ nature22327 (2017).

9. Poyner, D.R. et al. Pharmacol. Rev. 54, 233-246 (2002).

10. Khoshouei, M. et al. Nat. Commun. 7, 10534 (2016)

11. Danev, R., Tegunov, D. \& Baumeister, W. elife 6 e23006 (2017).

12. Thomsen, A.R. et al. Cell 166, 907-919 (2016).

13. Kumari, P. et al. Nat. Commun. 7, 13416 (2016).

14. Wehbi, V.L. et al. Proc. Natl. Acad. Sci. USA 110 1530-1535 (2013).

15. Shukla, A.K., Kumari, P., Ghosh, E. \& Nidhi, K. Methods Enzymol. 556, 549-561 (2015).

\title{
RNA base-pairing drives phase transitions
}

Repeat expansion disorders such as Huntington's disease, muscular dystrophy, amyotrophic lateral sclerosis (ALS) and frontotemporal dementia (FTD) are caused by an increase in the number of short nucleotide repeats within coding or noncoding regions of otherwise unrelated genes. A common pathological characteristic of these diseases is the formation of aberrant ribonucleoprotein (RNP) granules, which show features of phase-separated liquid-like compartments. Although numerous studies have characterized the ability of proteins to assemble these RNP granules, Ankur Jain and Ron Vale now demonstrate that sequence-specific multivalent base-pairing of RNAs can lead to phase separation, without necessarily requiring protein components (Nature http://dx.doi.org/10.1038/nature22386).

The authors show that RNAs with a critical number of CAG or CUG triplet repeats form viscous droplets in vitro (pictured). These can be dissolved by RNase A, monovalent cations and short complementary antisense oligonucleotides (ASOs), indicating that multivalent base-pairing and electrostatic interactions play a role in RNA clustering. CAG-repeat RNAs also form nuclear foci when expressed in cells. In contrast to their gel-like behavior in vitro, however, cellular foci show liquid-like properties. This may reflect the influence of cellular

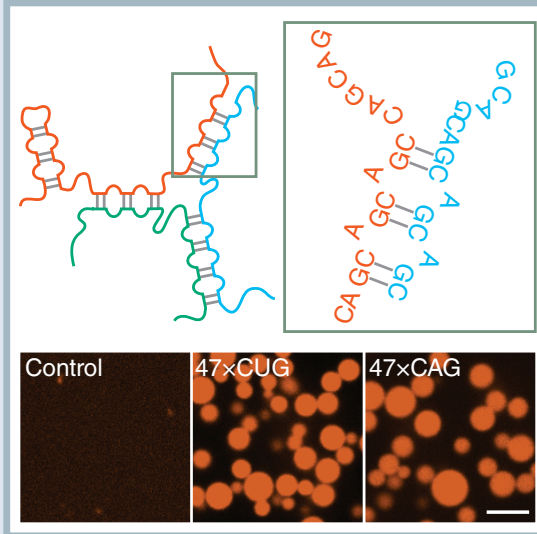
helicases that can remodel RNA base-pairing. The RNA foci colocalize with nuclear speckles, cellular compartments that are enriched in pre-mRNA splicing factors. They also sequester an endogenous RNA-binding protein, which has been implicated in repeat-expansion pathogenicity. Interestingly, agents that destabilize RNA base-pairing, such as ASOs or the nucleic acid intercalator doxorubicin, disrupt these cellular RNA foci without dissolving nuclear speckles. Guanine-rich nucleotide repeats that can form G-quadruplexes also show the propensity to form clusters, if the number of repeats is sufficient to induce multimolecular RNA interactions. This is particularly relevant because GGGGCC repeat expansion in the C9orf72 locus is one of the most common mutations associated with familial FTD and ALS.

Collectively, these data suggest that intermolecular base-pairing can trigger aggregation of RNA into nuclear foci, which may disrupt cellular homeostasis and contribute to disease-associated toxicity. While the precise pathological role of these foci remains to be established, it is curious that RNA foci and disease symptoms manifest only when the number of nucleotide repeats reach a certain threshold, an observation that may be related to the requirement of multivalency to promote phase transition. 